\title{
Prévision de l'endommagement ductile. Application à l'emboutissage de tôles minces
}

\author{
Mourad Khelifa a , Khémais Saanouni et Abel Cherouat \\ LASMIS-FRE 2719, Université de Technologie de Troyes, 12 rue Marie Curie, 10010 Troyes Cedex, France
}

Reçu le 13 mai 2005 ; accepté le 20 juin 2006

\begin{abstract}
Résumé - Ce travail est dédié à l'approche avancée pour modéliser et simuler un procédé de mise en forme de tôles minces par emboutissage dans l'objectif d'optimiser l'évolution de l'endommagement au cours du formage. La méthode proposée permet de prédire l'instant et l'endroit d'apparition de l'endommagement dans une pièce mécanique avant sa réalisation physique. Une approche locale basée sur le couplage fort entre le comportement élasto-plastique anisotrope à écrouissage mixte (isotrope et cinématique) non-linéaire et un endommagement ductile supposé isotrope est proposée. Les aspects numériques et théoriques des équations constitutives du modèle couplé seront présentés ainsi que la réduction des équations. La résolution globale du système d'équations est réalisée par un schéma dynamique explicite. La procédure est appliquée à la prévision de défauts dans un procédé d'emboutissage de type Swift. En dernier les résultats des simulations sont comparés à ceux de l'expérience.
\end{abstract}

Mots clés : Plasticité anisotrope / endommagement ductile / grandes déformations plastiques / contact et frottement / mise en forme / emboutissage / MEF

\begin{abstract}
Damage prediction in the deep drawing of thin sheets. This work deals with the study of advanced approach for modelling and the simulation in the deep drawing processes in order to optimize their technological parameters with respect to the damage occurrence. This allows to have coupled constitutive equations able to predict when and where micro defects can take place during the sheet deep drawing process. An advanced elastoplastic model based on mixed isotropic-kinematics hardening together with Hill type anisotropic plastic flow is fully coupled with isotropic ductile damage. First the fully coupled constitutive equations are summarized and their numerical aspects are discussed. The local integration procedure using the implicit scheme applied to a reduced number of constitutive equations is described. The global resolution procedure is also described using a dynamic explicit resolution strategy. This procedure has been applied to "optimize" various deep drawing processes with respect to damage occurrence. Finally an application is made to the simulation of the Swift deep drawing. A comparison with experimental results is also presented.
\end{abstract}

Key words: Plastic anisotropy / ductile damage / large plastic deformations / contact and friction / metal forming / deep drawing processes / FEM

\section{Introduction}

Le procédé d'emboutissage de tôles minces est largement utilisé dans l'industrie. Il consiste à déformer plastiquement, dans un plan, une tôle mince afin d'obtenir une pièce mécanique de forme géométrique plus ou moins complexe. La forme finale de la pièce est donnée par celles du poinçon et de la matrice. Ce procédé permet d'obtenir des pièces et des composants mécaniques dans de nombreux secteurs industriels comme la construction automobile (emboutissage de carter, de portières,

\footnotetext{
a Auteur correspondant : mourad.khelifa@utt.fr
}

de capots et autres éléments de carrosserie), la construction aéronautique, etc.

Longtemps l'emboutissage a été mis au point grâce à des procédures expérimentales basées sur le principe erreurs-corrections. Ce n'est que dans les années 1970 que la méthode des éléments-finis a commencé à être utilisée pour étudier numériquement l'emboutissabilité des tôles. Les simulations numériques doivent tenir compte du comportement anisotrope de tôles laminées à écrouissage nonlinéaire, de l'effet de grandes déformations plastiques et du contact/frottement entre la tôle et les outils (poinçon, serre-flan et matrice). 
Dans les années 1980 de nombreux logiciels de simulations numériques de l'emboutissage des tôles ont été mis sur le marché. Indéniablement, ces logiciels ont donné un nouvel essor à l'industrie de l'emboutissage de tôles minces et ont permis la fabrication de pièces de plus en plus complexes. Ces logiciels sont majoritairement basés sur la méthode incrémentale. Celle-ci consiste à discrétiser l'histoire du chargement en plusieurs incréments successifs et à prédire la forme finale de la pièce en partant de la connaissance du flan initial et de la forme des outils ([1] et [2]).

Malgré le développement de ces outils numériques de formage virtuel, un problème important n'a pas bénéficié d'un grand effort de développement. Il s'agit de la prévision de la limite de formage des tôles. En effet, on constate souvent l'apparition de fissures plus ou moins grandes dans les pièces formées. Ces fissures sont essentiellement dues à la déchirure ductile de la tôle dans les zones où elle atteint sa limite de déformation plastique (ductilité). Ce problème a été traité par la méthode dite des courbes limites de formage (CLF). Elle consiste à réaliser des essais simples de déformation plastique et à reporter sur un diagramme plan des déformations les valeurs limites des déformations principales pour lesquelles la rupture de l'éprouvette est observée [3]. On obtient ainsi une courbe limite de formage qui caractérise la limite de déformation de la tôle. Cette courbe supposée intrinsèque à chaque tôle est utilisée pour prévenir le risque d'apparition de défauts (fissures) dans un procédé de formage quelconque. Il suffit pour cela de simuler numériquement le procédé pour déterminer à chaque instant la distribution des déformations principales dans la tôle. Les points où les déformations limites (connues expérimentalement) sont atteintes sont supposés être le site d'une rupture locale [4]. Il est clair que cette méthode n'est pas de nature prédictive et ne peut servir utilement qu'à comparer des tôles entre elles en terme de propriétés de formage. En particulier, elle ne permet pas de prédire, au cours d'un calcul, où et quand une fissure (ou un défaut) peut apparaître dans la pièce.

Ce travail est justement consacré à cet aspect : proposer une méthodologie numérique pour prédire où et quand un endommagement ductile peut apparaître dans la pièce au cours de sa mise en forme ([5] et [6]).

L'identification des paramètres du modèle est faite sur des essais de traction d'une éprouvette en aluminium et sa validation sur un essai d'emboutissage de type Swift. Les résultats des simulations sont comparés à ceux de l'expérience.

\section{Modélisation plasticité-endommagement}

\subsection{Aspects théoriques}

La modélisation mécanique du comportement plastique endommageable est basée sur une approche thermodynamique des processus irréversibles avec variables internes. $\left(\underline{\varepsilon}^{\mathrm{e}}, \underline{\sigma}\right)$ : représentant le tenseur de déformations élastiques et le tenseur de contraintes de Cauchy; $(\underline{\alpha}, \underline{X})$ : représentant la contrainte cinématique; $(r, \bar{R})$ : représentant la contrainte isotrope et $(D, Y)$ : représentant l'endommagement ductile isotrope.

Les relations d'état sont définies dans [7] comme :

$$
\begin{aligned}
& \underline{\sigma}=(1-D) \underline{\underline{\Lambda}}: \underline{\varepsilon}^{\mathrm{e}} \\
& \underline{X}=\frac{2}{3} C(1-D) \underline{\alpha} \\
& R=(1-D) Q r \\
& Y=\frac{1}{2} \underline{\varepsilon}^{\mathrm{e}}: \underline{\underline{\Lambda}}: \underline{\varepsilon}^{\mathrm{e}}+\frac{1}{3} C \underline{\alpha}: \underline{\alpha}+\frac{1}{2} Q r^{2}
\end{aligned}
$$

$\Lambda$ est l'opérateur des propriétés élastiques d'ordre 4 . $C$ et $\overline{\bar{Q}}$ sont les paramètres d'écrouissage cinématique et isotrope respectivement.

La dissipation mécanique est décrite dans le cadre d'une théorie à une surface qui considère un seul potentiel de dissipation $F(\underline{\sigma}, \underline{X}, R, D)$, qui gouverne l'écoulement plastique $f(\underline{\sigma}, \underline{X}, \bar{R}, \bar{D})$ et l'évolution du dommage, et un seul multiplicateur plastique $\dot{\lambda}$ ([8] à [10])

$$
\begin{aligned}
& f(\underline{\sigma}, \underline{X}, R, D)=\frac{\|\underline{\sigma}-\underline{X}\|-R}{\sqrt{1-D}}-\sigma_{y}=0 \\
& F(\underline{\sigma}, \underline{X}, R, D)=f+\frac{3}{4} \frac{a}{C(1-D)} \underline{X}: \underline{X} \\
& +\frac{1}{2} \frac{b}{Q(1-D)} R^{2}+\frac{1}{(1-D)^{\beta}} \frac{S}{s+1}\left(\frac{Y-Y_{0}}{S}\right)^{s+1}
\end{aligned}
$$

avec $\|\underline{\sigma}-\underline{X}\|=\sqrt{(\underline{\sigma}-\underline{X}): \underline{\underline{H}}:(\underline{\sigma}-\underline{X})}$

$\sigma_{y}$ est la contrainte limite élastique; $a$ et $b$ sont les coefficients d'écrouissages non-linéaires cinématique et isotrope; $S, Y_{0}, \beta$ et $s$ caractérisent l'évolution de l'endommagement isotrope. $\underline{\underline{H}}$ est l'opérateur d'ordre 4 de Hill ([5], [11] et [12]) fonction de six constantes F, G, H, L, M et $N$ ([5] et $[13])$.

Basant sur la règle de la normalité, les relations complémentaires dérivent dans ([5] et [14]) comme suit :

$$
\begin{aligned}
& \underline{\dot{\varepsilon}}^{\mathrm{p}}=\dot{\lambda} \frac{\underline{\underline{H}}:(\underline{\sigma}-\underline{X})}{\sqrt{1-D}\|\underline{\sigma}-\underline{X}\|}=\frac{\dot{\lambda}}{\sqrt{1-D}} \underline{\tilde{n}} \\
& \dot{\alpha}=\underline{\dot{\varepsilon}}^{\mathrm{p}}-\dot{\lambda} a \underline{\alpha} \\
& \dot{r}=\dot{\lambda}\left[\frac{1}{\sqrt{1-D}}-b r\right] \\
& \dot{D}=\frac{\dot{\lambda}}{(1-D)^{\beta}}\left[\frac{Y-Y_{0}}{S}\right]^{\mathrm{s}}
\end{aligned}
$$

où $\underline{\tilde{n}}$ est la normale à la surface de charge. Le multiplicateur plastique est déterminé par la condition de consistance $f=\dot{f}=0$ donnée dans [14] :

$$
\dot{f}=\frac{2 \mu}{\sqrt{1-D}} \underline{\tilde{n}}: \underline{\dot{\varepsilon}}-h \dot{\lambda}=0
$$


où $h>0$ est le module tangent élastoplastique défini par :

$$
\begin{aligned}
h & =(2 \mu \sqrt{1-D}+C) \underline{\tilde{n}}: \underline{\tilde{n}}+Q-b \frac{R}{\sqrt{1-D}} \\
& -\frac{a}{\sqrt{1-D}} \underline{\tilde{n}}: \underline{X}+\frac{1}{(1-D)^{\beta+1}}\left[\frac{Y-Y_{0}}{S}\right]^{\mathrm{s}}\left[\frac{\sigma_{y}}{2}\right]
\end{aligned}
$$

Notons qu'en formulant le modèle ci-dessus en grandes déformations, nous avons utilisé une formulation en référentiel tournant. La rotation $Q$ de la configuration déformée est, à chaque instant $t$, solution d'une équation cinématique ([15] et $[16])$ :

$$
\underline{W}_{Q}=\underline{\dot{Q}} \underline{Q}^{T}, \quad \underline{Q}(t=0)=\underline{1}
$$

où 1 est le tenseur unite d'ordre 2 .

Les déformations élastiques sont supposées infinitésimales afin d'assurer l'additivité des taux de déformations

$$
\underline{D}=\underline{\dot{\varepsilon}}_{\mathrm{e}}^{\mathrm{J}}+\underline{D}^{\mathrm{p}}
$$

où $\dot{\varepsilon}_{\mathrm{e}}^{\mathrm{J}}$ est la dérivée rotationnelle de Jaumann du tenseur de petites déformations élastiques.

\subsection{Aspects numériques}

La résolution globale par la méthode des éléments-finis est basée sur la discrétisation spatiale des équations aux dérivées partielles du problème associées à des conditions initiales et aux limites données. Sous forme discrétisée, l'équilibre global du solide s'exprime par la fonctionnelle globale suivante :

$$
[M]\{\ddot{U}\}+\left\{F_{\text {int }}\right\}-\left\{F_{\text {ext }}\right\}=0
$$

où $[M]$ est la matrice masse, $\left\{F_{\text {int }}\right\}$ et $\left\{F_{\text {ext }}\right\}$ sont les vecteurs forces interne et externe respectivement.

Dans l'analyse Dynamique Explicite (DE), l'algorithme consiste à obtenir une solution de l'équation précédente à l'instant $t+\Delta t$ en fonction des quantités connues aux instants précédents

$$
\begin{aligned}
& \left\{\ddot{U}_{n}\right\}=[M]^{-1}\left[\left\{f_{\text {ext }}\right\}-\left\{f_{\text {int }}\right\}\right] \\
& \left\{\dot{U}_{n+1 / 2}\right\}=\left\{\dot{U}_{n-1 / 2}\right\}+\frac{\Delta t_{n+1}+\Delta t_{n}}{2}\left\{\ddot{U}_{n}\right\} \\
& \left\{U_{n+1}\right\}=\left\{U_{n}\right\}+\Delta t_{n+1}\left\{\dot{U}_{n+1 / 2}\right\}
\end{aligned}
$$

Notons que ce schéma explicite évite les itérations et le calcul de la matrice tangente. Sa stabilité et sa précision dépendent fortement du pas de temps $\Delta t[17]$.
La discrétisation des équations (7) à (10) est associée à une intégration asymptotique des équations (8) et (9). Les équations incrémentales sont :

$$
\begin{aligned}
\underline{D}_{n+1}^{\mathrm{p}} & =\underline{D}_{n}^{\mathrm{p}}+\frac{\Delta \lambda}{\sqrt{1-D_{n+1}}} \underline{\tilde{n}}_{n+1} \\
\underline{\alpha}_{n+1} & =\underline{\alpha}_{n} e^{-a \Delta \lambda}+\frac{\left(1-e^{-a \Delta \lambda}\right)}{a \sqrt{1-D_{n+1}}} \underline{\tilde{n}}_{n+1} \\
r_{n+1} & =r_{n} e^{-b \Delta \lambda}+\frac{\left(1-e^{-b \Delta \lambda}\right)}{b \sqrt{1-D_{n+1}}} \\
D_{n+1} & =D_{n}+\Delta \lambda\left[\frac{Y_{n+1}-Y_{0}}{S}\right]^{\mathrm{s}} \frac{1}{\left(1-D_{n+1}\right)^{\beta}}
\end{aligned}
$$

Notons qu'on doit intégrer les équations constitutives sur chaque incrément du temps afin de déterminer la valeur des variables $\underline{\sigma}_{n+1}, \underline{X}_{n+1}, R_{n+1}$ et $Y_{n+1}$.

L'intégration des équations constitutives permet de réduire l'ensemble des équations à intégrer de 15 à 8 ([6], [11] et [18]). Le système d'équations à résoudre est :

$$
\begin{aligned}
f_{n+1}\left(\Delta \lambda, \underline{\tilde{n}}_{n+1}, D_{n+1}\right)= & \frac{\left\|\underline{\sigma}_{n+1}-\underline{X}_{n+1}\right\|}{\sqrt{1-D_{n+1}}} \\
& -\frac{R_{n+1}}{\sqrt{1-D_{n+1}}}-\sigma_{y}=0
\end{aligned}
$$

$$
\begin{aligned}
\underline{h}_{n+1}\left(\Delta \lambda, \underline{\tilde{n}}_{n+1}, D_{n+1}\right) & =\underline{\underline{H}}:\left(\underline{\sigma}_{n+1}-\underline{X}_{n+1}\right) \\
& -\left\|\underline{\sigma}_{n+1}-\underline{X}_{n+1}\right\| \underline{\tilde{n}}_{n+1}=0
\end{aligned}
$$

$g_{n+1}\left(\Delta \lambda, \underline{\tilde{n}}_{n+1}, D_{n+1}\right)=D_{n+1}-D_{n}$

$$
-\Delta \lambda\left[\frac{Y_{n+1}-Y_{0}}{S}\right]^{\mathrm{s}} \frac{1}{\left(1-D_{n+1}\right)^{\beta}}=0
$$

avec :

$$
\begin{aligned}
& \left\|\underline{\sigma}_{n+1}-\underline{X}_{n+1}\right\|= \\
& \quad \sqrt{\left(\underline{\sigma}_{n+1}-\underline{X}_{n+1}\right): \underline{\underline{H}}:\left(\underline{\sigma}_{n+1}-\underline{X}_{n+1}\right)}
\end{aligned}
$$

$\underline{\sigma}_{n+1}=\underline{\sigma}_{n}+\left(1-D_{n+1}\right)\left[2 \mu \Delta \underline{\varepsilon}+\lambda_{\mathrm{e}} \operatorname{tr}(\Delta \underline{\varepsilon}) \underline{1}\right]$

$$
-2 G \sqrt{1-D_{n+1}} \Delta \lambda \underline{\tilde{n}}_{n+1}
$$

$$
\underline{X}_{n+1}=C\left(1-D_{n+1}\right)\left[\underline{\alpha}_{n} e^{-a \Delta \lambda}+\frac{\left(1-e^{-a \Delta \lambda}\right)}{a \sqrt{1-D_{n+1}}} \underline{\tilde{n}}_{n+1}\right]
$$

$$
R_{n+1}=Q\left(1-D_{n+1}\right)\left(r_{n} e^{-b \Delta \lambda}+\frac{\left(1-e^{-b \Delta \lambda}\right)}{b \sqrt{1-D_{n+1}}}\right)
$$

$G$ est le module de cisaillement (module d'incompressibilité); $\lambda_{\mathrm{e}}$ et $\mu$ sont les constantes de Lame.

Les inconnues du problème, équation (21), sont $\Delta \lambda$, $\underline{\tilde{n}}_{n+1}$ et $D_{n+1}$. La résolution du système d'équations se fait par l'algorithme de Newton-Raphson (voir [6] pour plus de détail). 


\subsection{Implémentation dans le code d'ABAQUS}

Pour le calcul des variables forces $\underline{\sigma}_{n+1}, \underline{X}_{n+1}, R_{n+1}$ et $D_{n+1}$ à l'instant $t_{n+1}$, nous avons utilisé l'algorithme de retour radial de Simo [18].

\section{(i) Prédiction élastique}

On se donne un incrément de déformations totales $\Delta \underline{\varepsilon}$ à l'instant $t_{n}$. La sollicitation est supposée purement élastique. La contrainte essai résultante à la fin de l'incrément est :

$$
\underline{\sigma}_{n+1}^{*}=\underline{\sigma}_{n}+\left(1-D_{n}\right)\left[2 \mu \Delta \underline{\varepsilon}+\lambda_{\mathrm{e}} \operatorname{tr}(\Delta \underline{\varepsilon}) \underline{1}\right]
$$

En substituant la valeur de $\underline{\sigma}_{n+1}^{*}$ dans l'expression de critère, on obtient :

$$
f_{n+1}^{*}=\frac{\left\|\underline{\sigma}_{n+1}^{*}\right\|-R_{n}}{\sqrt{1-D_{n}}}-\sigma_{y}
$$

avec

$$
\begin{gathered}
\left\|\underline{\sigma}_{n+1}^{*}\right\|=\sqrt{\underline{\sigma}_{n+1}^{*}: \underline{\underline{H}}: \underline{\sigma}_{n+1}^{*}} \\
\underline{X}_{n}=R_{n}=0
\end{gathered}
$$

Si $f_{n+1}^{*}<0$ la solution est purement élastique. La contrainte à l'instant $t_{n+1}$ est :

$$
\begin{aligned}
& \underline{\sigma}_{n+1}=\underline{\sigma}_{n+1}^{*} \\
& \Delta \lambda_{n+1}=\Delta \lambda_{n}, \underline{\tilde{n}}_{n+1}=\underline{\tilde{n}}_{n}, D_{n+1}=D_{n}
\end{aligned}
$$

\section{(ii) Correction radiale. Solution plastique endommageable}

Sinon si $f_{n+1}^{*}>0$, on utilise l'algorithme de NewtonRaphson pour calculer les inconnues $\Delta \lambda, \underline{\tilde{n}}$ et $D$ du système d'équations (Éq. (21)) :

$$
\left\{\begin{array}{l}
f_{n+1}\left(\Delta \lambda, \underline{\tilde{n}}_{n+1}, D_{n+1}\right)=0 \\
\underline{h}_{n+1}\left(\Delta \lambda, \underline{\tilde{n}}_{n+1}, D_{n+1}\right)=0 \\
g_{n+1}\left(\Delta \lambda, \underline{\tilde{n}}_{n+1}, D_{n+1}\right)=0
\end{array}\right.
$$

On fait des corrections sur les équations du problème (Éq. (21)) pour les ramener à zéro et on calcule à l'instant $t_{n+1}$ les inconnues $\Delta \lambda, \underline{\tilde{n}}$ et $D$. À convergence du système itératif de Newton-Raphson, on stocke la solution et on calcule les variables forces à l'instant $t_{n+1}$.

\section{Applications}

\subsection{Identification du modèle. Essai de traction}

Dans cette section on s'intéresse à l'identification de l'ensemble des paramètres du modèle de comportement élasto-plastique anisotrope fortement couplé à un endommagement ductile isotrope. L'identification consiste à minimiser la différence entre la courbe de référence (courbe expérimentale) et la courbe théorique obtenue par l'intégration numérique du modèle utilisé. Cela revient à déterminer un jeu de coefficients du modèle donnant une courbe théorique très proche de la réponse expérimentale.

Souvent, la résolution inverse pose des difficultés numériques (telle la non-unicité de la solution) liées au nombre important de constantes matérielles à déterminer. La procédure de caractérisation utilisée dans cette étude consiste initialement à identifier les paramètres de plasticité à une déformation plastique plus ou moins inférieure à $5 \%$ dans chaque direction d'anisotropie $\left(0^{\circ}, 45^{\circ}\right.$ et $90^{\circ}$ par rapport à la direction de laminage) sans tenir compte de l'effet de l'endommagement. Une fois les paramètres de plasticité déterminés, on identifie sur la totalité de la courbe pour déterminer les paramètres liés à l'endommagement tout en bloquant les paramètres d'écrouissage.

Pour l'identification du modèle, nous avons simulé les essais de traction sur des éprouvettes réelles en aluminium AL5754 par le logiciel ABAQUS/Explicit. Notons que les résultats expérimentaux sont fournis par le CETIM de Senlis.

Les caractéristiques géométriques de l'éprouvette de traction sont : épaisseur $e=1 \mathrm{~mm}$; hauteur $h=$ $12,52 \mathrm{~mm}$ et longueur initiale $l_{0}=80 \mathrm{~mm}$.

On s'intéresse à l'analyse de la partie utile de l'éprouvette. Le problème est similaire à celui d'une plaque rectangulaire soumise à ses extrémités à des conditions de déplacement bidirectionnel imposé.

L'élément d'analyse choisi dans la bibliothèque d'ABAQUS/Explicit est un élément hexaédrique en 3D à huit nœuds de type C3D8R utilisant une intégration réduite. Un maillage de 2366 éléments : 13 éléments suivant la hauteur, 91 éléments suivant la longueur et deux couches d'éléments dans l'épaisseur de l'éprouvette est utilisé. Les simulations sont faites avec une vitesse de traction $v=10 \mathrm{~mm} . \mathrm{s}^{-1}$ et une densité $\rho=2700 \mathrm{~kg} . \mathrm{m}^{-3}$. Les coefficients du modèle sont ceux rassemblés dans le tableau 1.

La distribution de champs mécaniques (déformation plastique cumulée et endommagement) pour certains déplacements, suivant la direction de laminage $0^{\circ}$, sont donnés sur les courbes de la figure 1a.

Pour la direction de laminage $0^{\circ}$, on distingue clairement les trois stades d'évolution de l'endommagement. La déformation est homogène jusqu'à un déplacement inférieur à $u=16,2 \mathrm{~mm}$. Au-delà de cette limite la déformation devient hétérogène. On constate un début de localisation diffuse pour un déplacement de l'ordre de 18,6 $\mathrm{mm}$. Une striction localisée sous la forme de deux bandes de cisaillement apparaît rapidement et une fissure macroscopique s'amorce à l'intersection des deux bandes pour $u=21 \mathrm{~mm}$.

Pour la direction $45^{\circ}$ et $90^{\circ}$, on se contente de représenter l'évolution de la déformation plastique cumulée et de l'endommagement à la rupture.

Suivant les trois directions, on note que les champs mécaniques (déformation plastique cumulée et endommagement) passent par les trois stades d'évolution : champs 
Tableau 1. Caractéristiques mécaniques du matériau AL 5754.

\begin{tabular}{lllllll}
\hline \multirow{2}{*}{ Élasticité } & $E[\mathrm{GPa}]$ & \multicolumn{5}{c}{$\nu$} \\
& 70, & \multicolumn{5}{c}{0,3} \\
\hline \multirow{3}{*}{ Plasticité } & $\sigma_{y}[\mathrm{MPa}]$ & $C[\mathrm{GPa}]$ & $a$ & \multicolumn{2}{c}{$Q[\mathrm{MPa}]$} & $b$ \\
& 114, & 2,1 & \multicolumn{2}{c}{21,} & 554,3 & 3,65 \\
& $\mathrm{~F}$ & $\mathrm{G}$ & $\mathrm{H}$ & $\mathrm{L}$ & $\mathrm{M}$ & $\mathrm{N}$ \\
& 0,9 & 0,6 & 0,35 & 1,5 & 1,5 & 1,65 \\
\hline \multirow{2}{*}{ Endommagement } & $S[\mathrm{MPa}]$ & \multicolumn{2}{c}{$s$} & & \multicolumn{3}{c}{$Y_{0}[\mathrm{MPa}]$} \\
& 13, & \multicolumn{2}{c}{11,} & & 9, & \multicolumn{2}{c}{0,1} \\
\hline
\end{tabular}

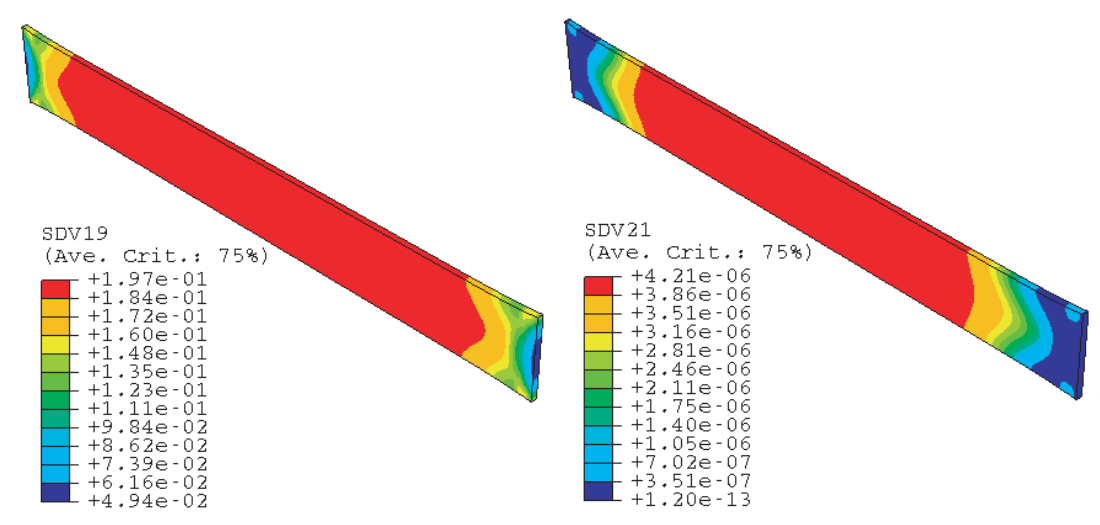

Déformation plastique cumulée

Endommagement

(a) : Déplacement $u=16.2 \mathrm{~mm}$

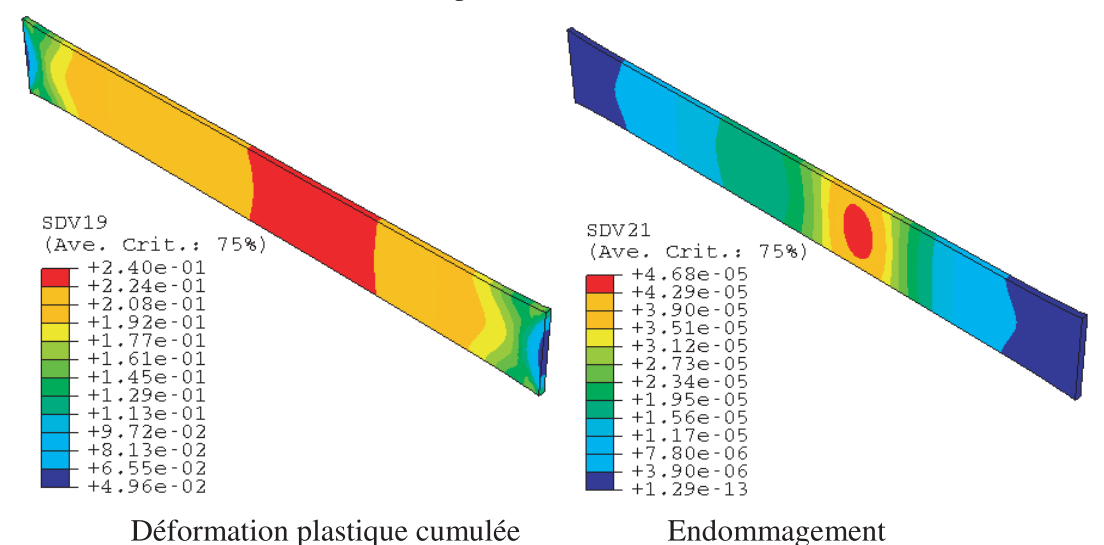

(b) : Déplacement $\mathrm{u}=18.6 \mathrm{~mm}$



(c) : Déplacement u = $21 \mathrm{~mm}$

Fig. 1a. Isovaleurs de la déformation plastique cumulée et de l'endommagement suivant la direction de laminage $0^{\circ}$. 


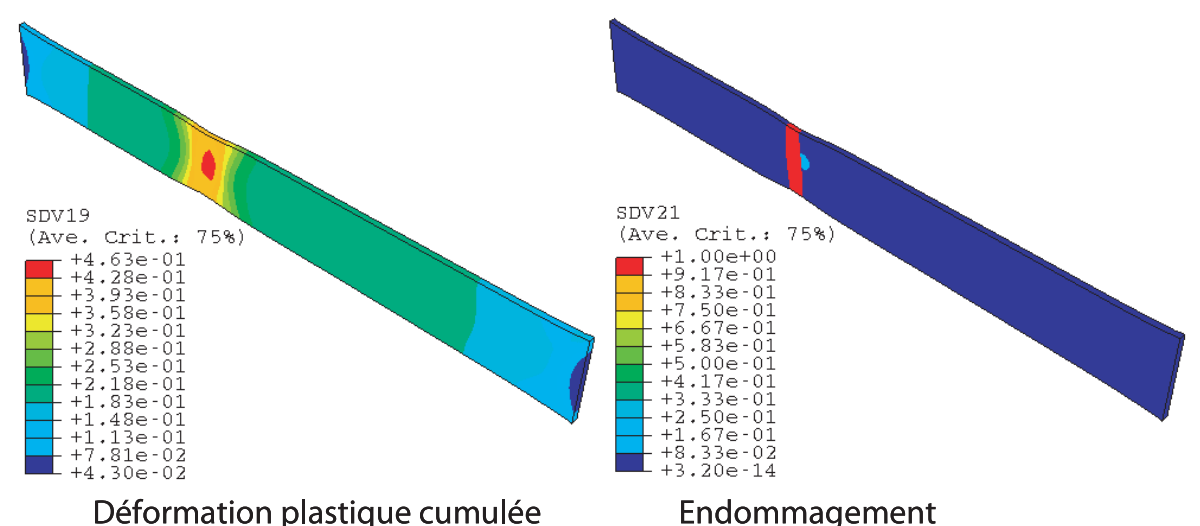

(a) : Déplacement u $=20.4 \mathrm{~mm}$

Fig. 1b. Isovaleurs de la déformation plastique cumulée et de l'endommagement suivant la direction $45^{\circ}$.

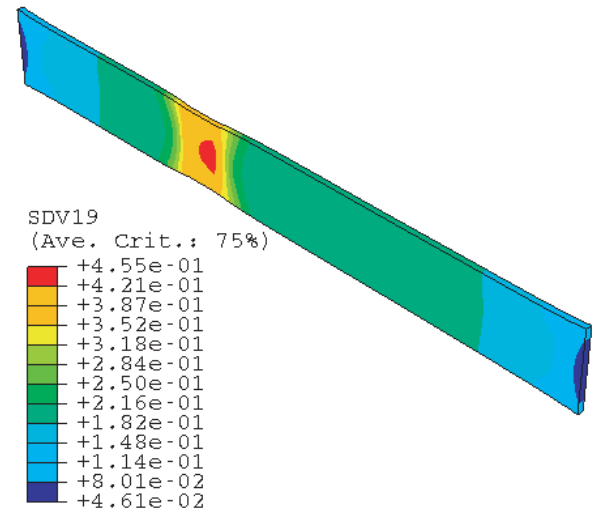

Déformation plastique cumulée

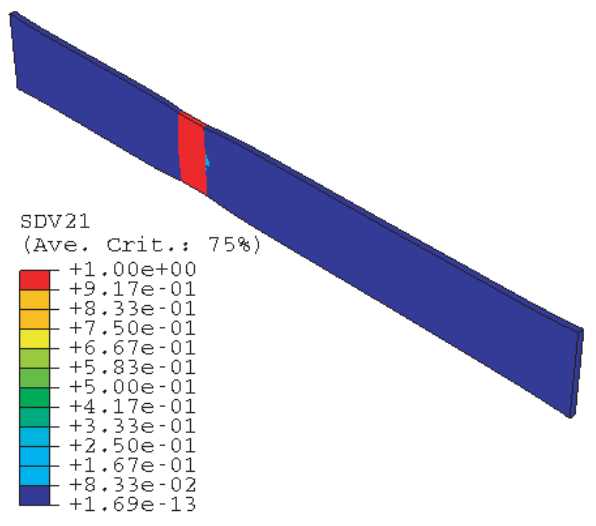

Endommagement

(a) : Déplacement $\mathrm{u}=22.8 \mathrm{~mm}$

Fig. 1c. Isovaleurs de la déformation plastique cumulée et de l'endommagement suivant la direction transverse $90^{\circ}$.

homogènes, striction diffuse et striction localisée. Les figures $1 \mathrm{a}-\mathrm{c}$ montrent que la rupture est perpendiculaire à l'axe principal de l'éprouvette et ce pour les trois directions $0^{\circ}, 45^{\circ}$ et $90^{\circ}$.

Les résultats des simulations effort-déplacement en chaque direction $0^{\circ}, 45^{\circ}$ et $90^{\circ}$ sont comparés à l'expérience (Fig. 2). On constate sur les figures $2 \mathrm{a}-\mathrm{c}$ que les résultats de la simulation approchent ceux de l'expérience jusqu'à un déplacement de l'ordre de $6 \mathrm{~mm}$. La rupture de l'éprouvette dans la direction $0^{\circ}$ et $45^{\circ}$ n'est pas bien prévue comparativement à la direction transverse $90^{\circ}$. Ceci peut être dû au fait que l'endommagement considéré est isotrope.

Le tableau 2 résume les valeurs du déplacement à la rupture et de l'effort maximal enregistré pour chaque direction $0^{\circ}, 45^{\circ}$ et $90^{\circ}$. Le tableau 2 montre que l'effort maximal ainsi que le déplacement à la rupture sont mieux approchés suivant les directions $45^{\circ}$ et $90^{\circ}$.

Après identification des paramètres du modèle couplé, on utilise le même matériau AL5754 pour simuler un procédé d'emboutissage.

\subsection{Simulation d'emboutissage de Swift}

Dans cette section, la procédure implémentée dans ABAQUS/Explicit s'applique à la prévision de l'endommagement ductile dans un procédé d'emboutissage de type Swift réalisé au CETIM/Senlis. Le flan est une tôle de diamètre $73 \mathrm{~mm}$ et d'épaisseur $1 \mathrm{~mm}$ (Fig. 3). Le flan est maillé en utilisant des éléments solides C3D8R. Les outils indéformables (matrice, poinçon et serre-flan) sont discrétisés avec des éléments de coque rigides C3D4. La vitesse du poinçon est $v=10 \mathrm{~mm} . \mathrm{s}^{-1}$. Le coefficient de frottement de Coulomb pris pour l'ensemble des corps en contact est $\eta=0,17$.

La figure 4 rassemble quelques distributions de champs mécaniques (déformation plastique cumulée et endommagement) pour certaines valeurs de déplacement du poinçon.

On observe un début de localisation à un déplacement du poinçon $u=7,84 \mathrm{~mm}$. La localisation apparaît sur une zone circulaire épousant la forme du poinçon. Au fur et à mesure que le déplacement du poinçon augmente les valeurs de l'endommagement augmentent dans la zone circulaire avec un diamètre décroissant. Une forte localisation est enregistrée à un déplacement du poinçon 


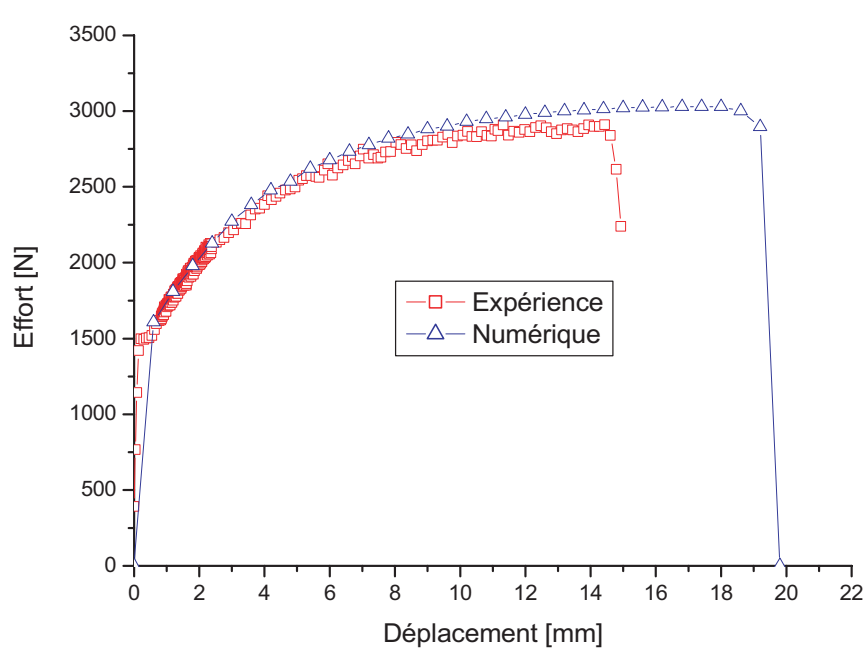

(a) : Direction de laminage $0^{\circ}$

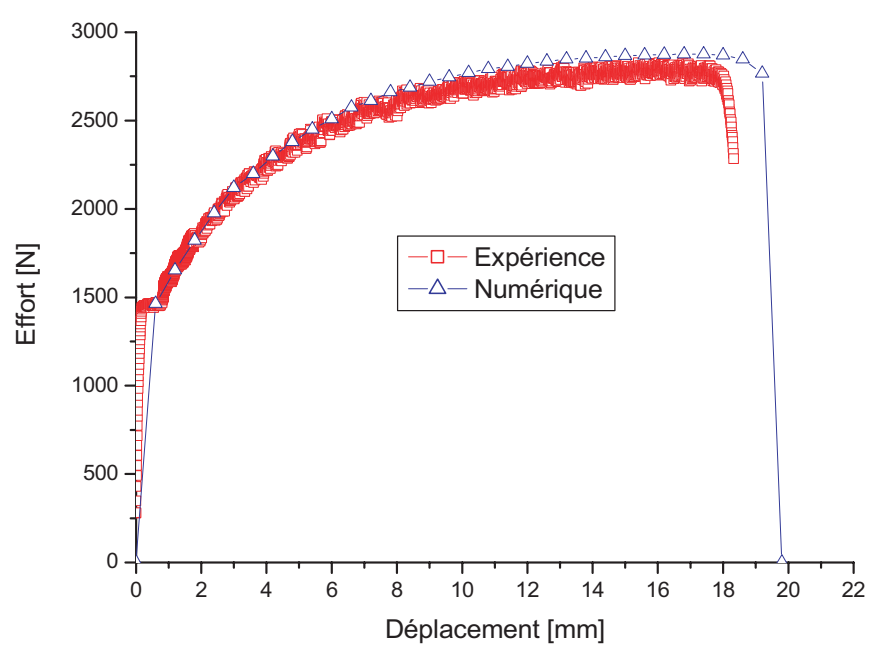

(b) : Direction $45^{\circ}$

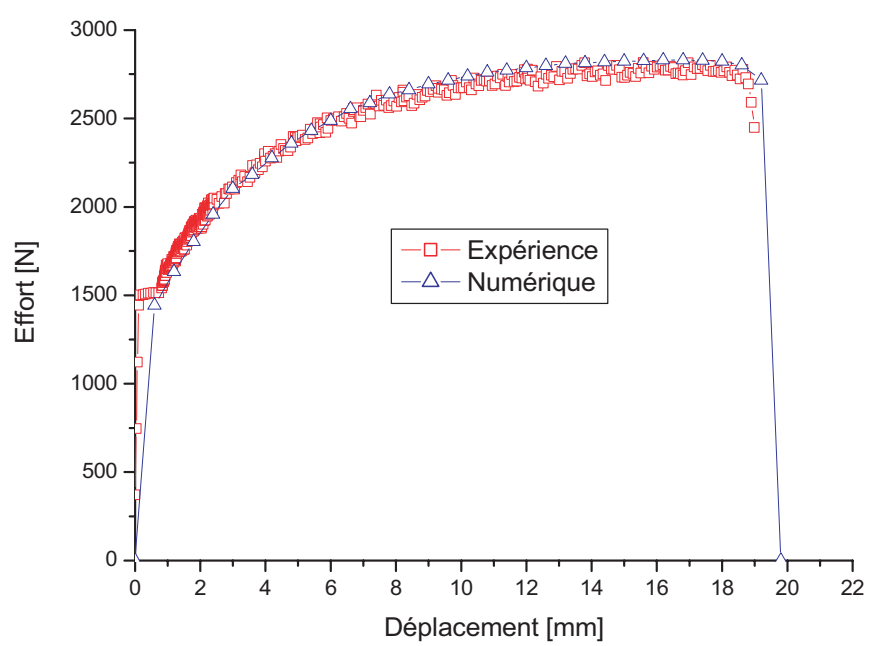

(c) : Direction transverse $90^{\circ}$

Fig. 2. Courbes effort-déplacement.



Fig. 3. Emboutissage de Swift.

$u=10,28 \mathrm{~mm}$ qui se transforme rapidement en une fissure macroscopique pour $u=11,03 \mathrm{~mm}$.

Les figures $5 \mathrm{a}$ et $\mathrm{b}$ montrent une comparaison entre la rupture expérimentale et celle prédite par notre méthodologie.

Dans un essai d'emboutissage, le risque de rupture est évalué en utilisant la courbe limite de formage FLC définie par le plan de déformations principales (Fig. 6). Les champs de déformations à différentes profondeurs du poinçon sont mesurés pour estimer l'évolution des déformations dans des zones à risque d'apparition de striction.

Les points expérimentaux et numériques sont relevés dans la zone encadrée de l'embouti (voir la photo dans la Fig. 6). Les points situés au-dessus de la FLC représentent le site de la rupture de la tôle.

La figure 7 présente la comparaison entre les résultats expérimentaux et numériques en terme de l'effortdéplacement du poinçon. L'effort maximal obtenu par la simulation $F=25,93 \mathrm{kN}$ approche celui de l'expérience $F=25,92 \mathrm{kN}$. L'instant de la rupture donné par la simulation est identique à celui obtenu expérimentalement.

\section{Conclusion et perspectives}

Une méthodologie prédictive de l'amorçage d'une fissure macroscopique a été proposée et appliquée à l'emboutissage de pièces minces orthotropes. La méthode expérimentale CLF est un post-traitement à la fin de simulation qui néglige l'interaction entre plasticité et endommagement. Dans le cas de notre démarche, le mode couplé plasticité-endommagement s'avère indispensable pour considérer les effets de couplage et éviter la surestimation de la formabilité. La présente procédure permet de prédire l'avènement de la rupture ductile lors d'un emboutissage de tôles minces. Les résultats des simulations numériques sur l'ensemble des essais, traction 
Tableau 2. Valeurs des déplacements et des efforts maximums à la rupture.

\begin{tabular}{lcccccc} 
& $0^{\circ}$ & \multicolumn{3}{c}{$45^{\circ}$} & \multicolumn{3}{c}{$90^{\circ}$} \\
\cline { 2 - 7 } & $u[\mathrm{~mm}]$ & $F[\mathrm{daN}]$ & $u[\mathrm{~mm}]$ & $F[\mathrm{daN}]$ & $u[\mathrm{~mm}]$ & $F[\mathrm{daN}]$ \\
\hline Expérience & 14,97 & 290,5 & 18,37 & 281,6 & 19,02 & 279,8 \\
Numérique & 19,88 & 302,3 & 19,62 & 286,6 & 19,78 & 284,2 \\
Erreur [\%] & 32,79 & 4,06 & 6,81 & 1,77 & 3,98 & 1,57 \\
\hline
\end{tabular}

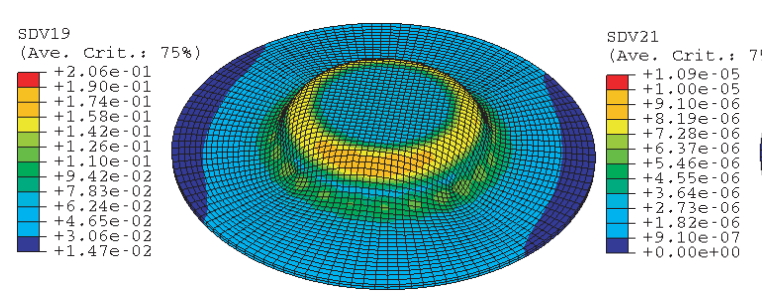

Déformation plastique cumulée

(a) : Déplacement $\mathrm{u}=7.84 \mathrm{~mm}$

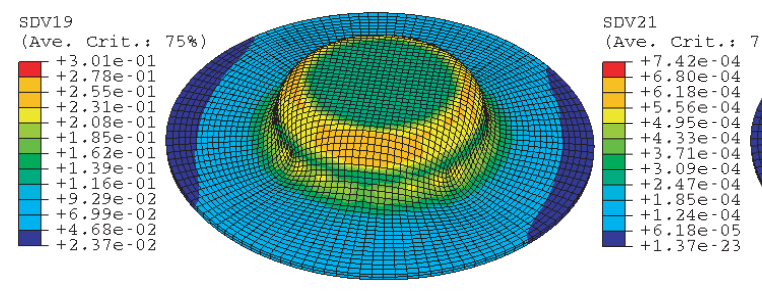

Déformation plastique cumulée

(b) : Déplacement $u=10.28 \mathrm{~mm}$

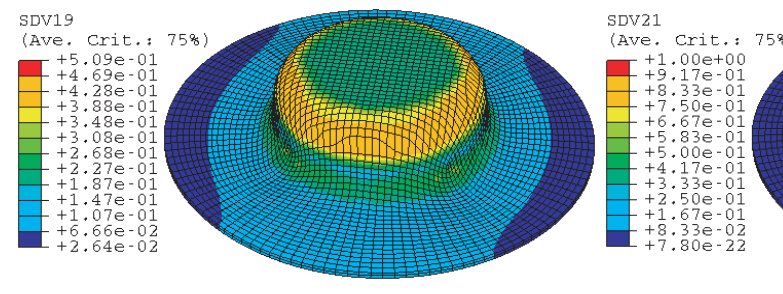

Déformation plastique cumulée

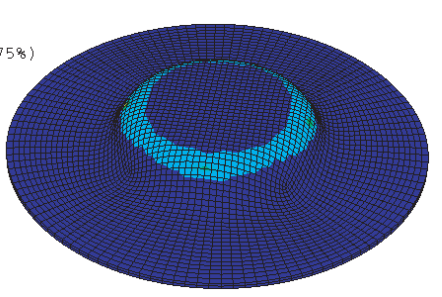

Endommagement

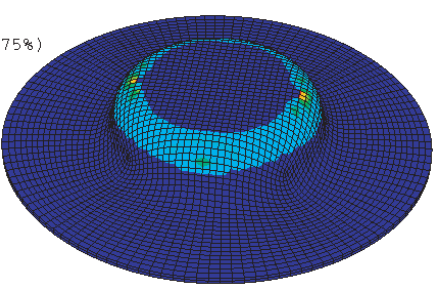

Endommagement

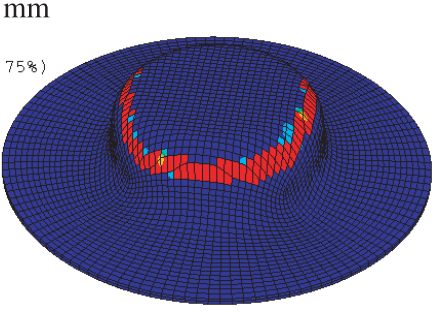

Endommagement (c) : Déplacement $\mathrm{u}=11.03 \mathrm{~mm}$

Fig. 4. Isovaleurs de la déformation plastique cumulée et de l'endommagement pour certains déplacements du poinçon.

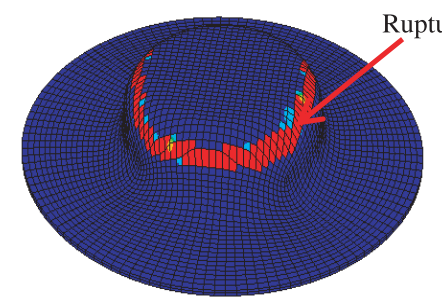

(a) : Endommagement



(b) : Expérience
Fig. 5. Apparition de la rupture sur l'embouti.

et emboutissage, sont en bon accord avec les résultats expérimentaux obtenus par le CETIM/Senlis (France).

Plusieurs points sont envisagés pour enrichir la méthodologie prédictive et mettre à la disposition de l'ingénieur un véritable outil de formage virtuel : formulation non-locale ou en gradient de dommage pour s'affranchir de la dépendance vis-à-vis du maillage; formulation à deux surfaces pour mieux décrire l'anisotropie

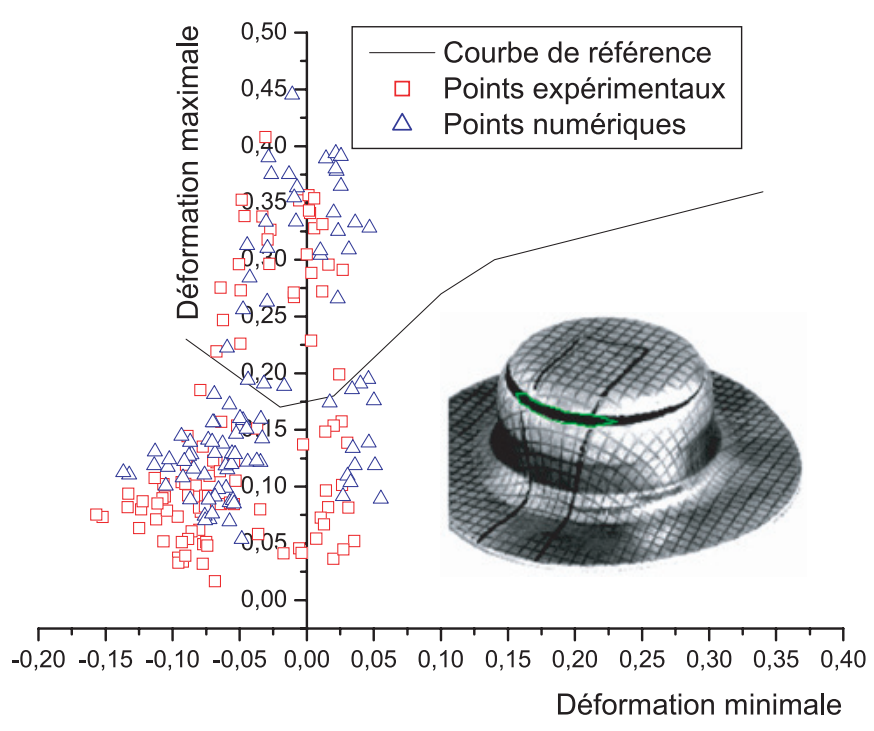

Fig. 6. Courbes Limites de Formage CLF. 


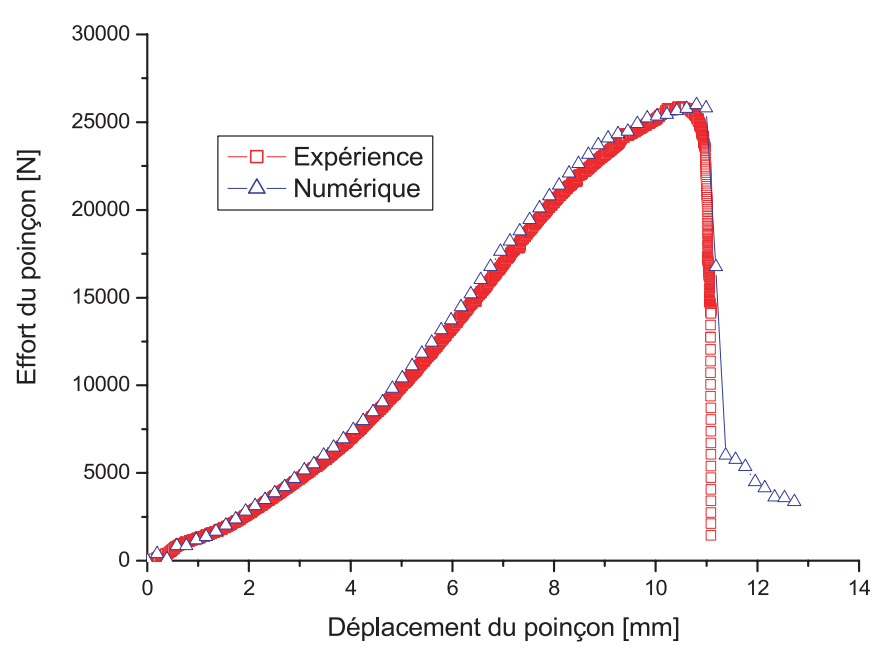

Fig. 7. Courbes effort-déplacement.

de l'endommagement; utiliser des modèles micro-macro seuls capables de traduire naturellement les évolutions microstructurales des matériaux subissant de larges déformations plastiques, etc.

Remerciements. Cette étude se déroule dans le cadre d'un contrat CPER intitulé «Simul_Endo ». Les auteurs remercient l'État et la Région Champagne-Ardenne pour leur soutien financier ainsi que la collaboration du CETIM/Senlis.

\section{Références}

[1] E. Onate, J. Rojek, C. Garcia Garino, NUMISTAMP: a research project for assessment of finite-element models for stamping processes, J. Mat. Processing Technology 50 (1995) 17-38

[2] M.L. Boubakar, Ph. Boisse, J.C. Gelin, Numerical implementation of orthotropic plasticity for sheet-metal forming analysis, J. Mat. Processing Technology 65 (1997) $143-152$

[3] H. Sfar, A. Maillard, Applications de la simulation de l'emboutissage, CETIM, Rap. $\mathrm{N}^{\circ}$ 1B8422, décembre 2000

[4] H. Sfar, A. Maillard, Aides à la réalisation de l'emboutissage : simulation et mesure automatique des déformations, CETIM, Recueil de conférences de la journée d'information du 17 novembre 1998

[5] M. Khelifa, H. Badreddine, N. Belamri, M.-A. Gahbiche, K. Saanouni, A. Cherouat, A. Dogui, Effect of anisotropic plastic flow on the ductile damage evolution in sheet metal forming, Application to the circular bulging test, Inter. J. Forming Processes 8 (2005)

[6] M. Khelifa, Simulation numérique de l'endommagement en formage des structures minces, thèse de Doctorat, Université de Technologie de Troyes, France, 2004

[7] K. Saanouni, C. Forster, F. Ben Hatira, On the inelastic flow with damage, Int. J. Damage Mechanics 3 (1994) 140-169

[8] J.-L. Chaboche, Le concept de contrainte effective appliqué à l'élasticité et à la viscoplasticité en présence d'un endommagement anisotrope, in J.-P. Boehler (éd.) Colloque EuroMech 115, Grenoble, Éditions du CNRS, 1979, pp. 737-760

[9] J. Lemaitre, A Continuum damage mechanics model for ductile fracture, J. Eng. Mat. Tech. 107 (1985) 83-89

[10] J. Lemaitre, A course on damage mechanics, SpringerVerlag, 1996

[11] J.-L. Chaboche, G. Cailletaud, Integration methods for complex plastic constitutive equations, Computer methods in applied mechanics and engineering 133 (1996) $125-155$

[12] R. Hill, A theory of yielding and plastic flow of anisotropic metals, Royal Soc., London Proc., 1948, p. 281

[13] J. Lemaitre, J.L. Chaboche, Mécanique des matériaux solides, Édition Dunod, 1988

[14] K. Saanouni, J.-L. Chaboche, Computational Damage Mechanics, Application to Metal Forming, Chapter 7 of the volume 3: Numerical and Computational methods, $\mathrm{R}$. de Borst, H.A. Mang Ed., in Comprehensive Structural Integrity, I. Milne, R.O. Ritchie, B. Karihaloo (éd.), ISBN: 0-08-043749-4, 2003

[15] F. Sidoroff, The geometrical concept of intermediate configuration and elastic-plastic finite strain, Arch. Mech. 25 (1973) 299-308

[16] A. Dogui, Plasticité anisotrope en grandes déformations, thèse de doctorat d'état, Université Claude Bernard, Lyon I, 1989

[17] ABAQUS, Theory manual, version 6.2, Hibbit, Karson and Sorensen, Inc., 2001

[18] J.C. Simo, T.J.R. Hughes, Computational inelasticity, Springer, New York, 1998 